\title{
New Brucella abortus S19 Mutant to Improve Distinction Between Infected and Vaccinated Animals
}

\author{
Bahar Nayeri Fasaei ${ }^{1,}$, Soulmaz Naserli ${ }^{1}$, Taghi Zahraei Salehi ${ }^{1}$, Alireza Saeedinia ${ }^{2}$, Alimohammad \\ Behroozikhah $^{3}$, Iradj Ashrafi Tamai ${ }^{1}$
}

${ }^{1}$ Department of Microbiology, Faculty of Veterinary Medicine, University of Tehran, Tehran, Iran

${ }^{2}$ Department of Bioscience and Biotechnology, Malek-Ashtar University of Technology, Tehran, Iran

${ }^{3}$ Department of Brucellosis, Razi Vaccine and Serum Research Institute, Agricultural Research Education and Extension Organization (AREEO), Karaj, Iran

* Corresponding author: Bahar Nayeri Fasaei, Department of Microbiology, Faculty of Veterinary Medicine, University of Tehran, Tehran, Iran. Tel: +98-2161117050; Fax: +98-2166431105; E-mail: nayerib@ut.ac.ir

\begin{abstract}
Background: Using Brucella abortus Strain 19 (S19) to control bovine brucellosis is restricted due to induce antibodies to the O-side chain of the smooth lipopolysaccharide (LPS) which may be difficult to differentiate vaccinated and infected animals. Furthermore, it is virulent for humans and can induce abortion to cattle.

Objectives: The aim of this study was to employ gene knockout B. abortus S19 for the first time to eliminate diagnostic defects and obtain the attenuated mutant strain.

Material and Methods: The $w b k A$ gene, which is one of the LPS O-chain coding genes, was knocked out in vaccinal Brucella abortus S19. The proliferative response and immunoglobulin M production were analyzed in $w b k A$ deletion strain-infected BALB/c mice.

Results: The loss of $w b k A$ gene function resulted in induction of the splenocyte proliferative response in mice infected by the mutant S19 strain compare to those induced by parental S19 and RB51 strains. Moreover, wbkA mutant did not induce any IgM antibody response using the enzyme-linked immunosorbent assay.

Conclusions: As a result, the new mutant S19 strain had deficiency in its LPS O-chain structure, besides cannot induce IgM response then, reduce mistakes to discriminate between vaccinated and infected animal, and also can be considered as a new vaccine candidate.
\end{abstract}

Keywords: Brucella abortus; Mutant Strains; Gene Knockout Techniques

\section{Background}

Brucella abortus causes serious chronic infections in livestock and humans (1). It is a facultative intracellular pathogen, which survives in a variety of cells, including phagocytic and non-phagocytic host cells. Its virulence and ability to cause chronic infections is thought to be due to its ability to avoid the killing mechanisms within macrophages (2). LPS is a major component of the outer membrane of Gram-negative bacteria and one of the Brucella main virulence factors, considered to be important for immune evasion during early stages of Brucella infection (3). LPS is synthesized as two separate components, the lipid A/core oligosaccharide and $\mathrm{O}$ antigen synthesized on a lipid carrier by enzymes encoded by the $w b o / w b k$ gene cluster (4). This cluster contains two regions. The region wbo encodes two putative glycosyltransferases ( $w b o A$ and $w b o B)$, and the region $w b k$ contains genes putatively involved in perosamine synthesis (gmd and per), its formylation $(w b k C)$ and polymerisation (catalised by glycosyltransferases) ( $w b k A$ and $w b k E$ ), as well as those for bactoprenol priming ( $w b k D$ and $w b k F)$ and Opolysaccharide translocation ( $w z m$ and $w z t)(5)$.

Currently, there are two main strains used as live attenuated B. abortus vaccines, strain 19 (S19, smooth) and RB51 (rough) (6). The use of the current vaccine strain, B. abortus S19, in the eradication of bovine brucellosis is limited because field-infected animals cannot be reliably distinguished from vaccinated animals. Moreover, B. abortus S19 is virulent for humans and cattle (7). On the other hand, RB51 is an attenuated, stable, rough mutant of B. abortus 2308 (8). One of the factors contributing to its rough phenotype 
is the interruption, by an insertion sequence (IS711) element, of the $w b o A$ gene encoding a glycosyltransferase essential for the $\mathrm{O}$-side chain synthesis and mutation in more than one of the genes necessary for the expression of a smooth phenotype (9). As a rough mutant, strain RB51 does not antibody formation to the O-side chain of smooth LPS of $B$. abortus, thereby enabling clear serological differentiation between vaccinated and infected animals (10). This strain is currently used as the official vaccine against cattle brucellosis in some countries $(6,9)$. The results show that RB51 is more attenuated in mice than S19, with shorter persistence times even at a 100 fold higher dose (10). However, B. abortus RB51 has a limitation because it is resistant to rifampicin, an antibiotic of choice for brucellosis treatment in pregnant women, children, and brucellosis endocarditis cases. Besides, depending upon the dosage and route of delivery, its effectiveness for prevention of abortion is variable (9). Therefore, efforts have been focused on searching for a better live rough attenuated vaccine that would be capable of inducing efficient cellular immunity and protection (10). In B. abortus, a wbkA-homologous gene was predicted to be absolutely required for the $\mathrm{O}$ side chain production. The WbkA protein can interact with WboA to elongate the Brucella LPS O-sidechain by $\alpha-1,2$ and $\alpha-1,3$ links (11).

\section{Objectives}

In this study, we used Brucella S19 wbkA mutant as constructed previously (12) to study the role of the gene in lymphocyte stimulation as an indicator of cellular immunity and immunoglobulin $\mathrm{M}(\operatorname{IgM})$ secretion as an interfering antibody in mice.

\section{Materials and Methods}

\subsection{Infection of Mice and Serum Antibody Response Assay}

Three groups of 15 female 6-weeks-old BALB/c mice (Razi Vaccine and Serum Research Institute) were used. They were blind randomly distributed and accommodated in the animal facility of Tehran University. Mice were injected intraperitoneally $2 \times 10^{6}$ colony-forming units of B. abortus RB51, S19 and $\triangle w b k A$ in $0.2 \mathrm{~mL}$ of phosphate-buffered saline (PBS, $\mathrm{pH}$ 7.2). The fourth group of mice was injected PBS as the negative control (13). Serum IgM titers in the immunized mice were determined by sandwich enzymelinked immunosorbent assay (ELISA) on day 0, 7, 14 and 21 post-inoculation. IgM isotype was determined using a commercial ELISA kit (Biospes, China) following the manufacturer's instructions. The plate was read at a wavelength of $540 \mathrm{~nm}$ (Stat Fax ${ }^{\otimes} 2100$, Awareness Technologies, USA).

\subsection{Lymphocyte Culture and MTT Assay}

On days $0,7,14,21$ and 28 after the inoculation, the mice were sacrificed, and the spleens were extracted for lymphocyte cultures. The spleens from each mouse was placed in a Petri dish containing $5 \mathrm{~mL}$ of RPMI-1640 (Gibco, USA) medium supplemented with $100 \mathrm{U} / \mathrm{mL}$ of penicillin and $100 \mu \mathrm{g} / \mathrm{mL}$ of streptomycin. The spleen was mashed with the plunger of a syringe. The cellular suspension was gently layered on FicollHypaque (Baharafshan, Iran) and isolated by centrifugation, followed by centrifugation at $1,600 \mathrm{rpm}$ for $20 \mathrm{~min}$. peripheral blood mononuclear cells (PBMC's) were carefully aspirated from the plasma Ficoll interface and washed three times with RPMI1640 medium using centrifugation at $1,600 \mathrm{rpm}$ for 10 min each (Eppendorf, Germany). Cell viability was checked by trypan blue staining, and the cells were counted using a hemocytometer as per standard procedure. The cells were finally suspended in RPMI1640 supplemented with $10 \%$ fetal calf serum (FCS, Gibco, USA). The counted cells were plated into flatbottom 96-wells tissue culture plates. In order to compare PBMC proliferation, we used MTT Cell Proliferation Kit I (Roche, Germany). Approximately $10^{5}$ cells/well were cultured in RPMI-1640 medium supplemented with $10 \%$ of FCS, $1 \%$ of L-glutamine, $1 \%$ of 4-(2-hydroxyethyl)-1-piperazineethanesulfonic acid (HEPES), $0.1 \%$ of 2 -mercaptoethanol and $0.1 \%$ of penicillin/streptomycin for $96 \mathrm{~h}$ at $37^{\circ} \mathrm{C}, 5 \% \mathrm{CO}_{2}$. After the incubation, $10 \mu \mathrm{L}$ of the 3-(4,5-dimethylthiazol-2yl)-2,5-diphenyltetrazolium bromide (MTT) labeling reagent was added to each well. The cells were incubated with MTT for $4 \mathrm{~h}$ in a humidified atmosphere of $5 \% \mathrm{CO}_{2}$ at $37^{\circ} \mathrm{C}$. After the reaction, $100 \mu \mathrm{L}$ of the solubilisation solution was added, and the plates were incubated overnight in a humidified atmosphere of $5 \% \mathrm{CO}_{2}$ at 37 ${ }^{\circ} \mathrm{C}$ to solubilize the formazan salt crystals. The soluble formazan product was spectrophotometrically quantified at a wavelength of $540 \mathrm{~nm}$ using a microplate reader (Stat $\mathrm{Fax}^{\otimes} 2100$, Awareness Technologies).

The statistical analysis was carried out using SPSS software, v.19 (SPSSInc, USA) and Microsoft Excel 2010 (Microsoft corp.). For determination of statistical significance between experimental groups a student's $T$ test was performed on data. The $\mathrm{P}$ value $<0.05$ is considered significant.

\section{Results}

4.1. Antibody Response Against B. abortus S19 $\triangle w b k A / k a n^{R}$

The sera collected on days $0,7,14$ and 21 postvaccination were used to detect the presence of specific antibodies by ELISA. The levels of serum IgM able to react with a purified anti-Brucella IgM antibody in a sandwich ELISA were evaluated. Immunization with the $w b k A$ mutant did not induce an antibody response with IgM isotypes. A similar distribution occurred in the sera of the mice inoculated with the RB51 strain; however, 
the IgM levels increased in the mice vaccinated with S19 on days 14 and 21 post-injection. This indicates that on days 14 and 21 post injection, the IgM titer was higher in the mice immunized with $B$. abortus S19 than in those immunized with B. abortus S19 $\triangle$ wbkA and RB51 (Fig. 1).

\subsection{Cell Proliferation}

To investigate the cellular immune response induced by the $\Delta w b k A$ strain, we analyzed proliferative splenocyte responses of the mice vaccinated with $B$. abortus S19, RB51 and the mutant strain. The data obtained were expressed as the 'mean plus two standard deviations'. The result of the MTT assay shown in Figure 2 demonstrate that the highest proliferative activity was observed in the mice vaccinated with S19, followed by those vaccinated with the mutant and RB51.

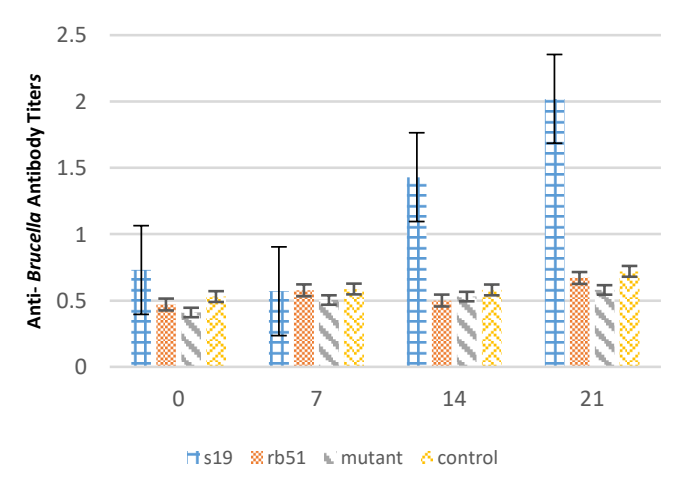

Figure 1. Average optical density obtained in the ELISA assay performed to evaluate immune responses in the mice during three weeks after the immunization with Brucella abortus S19, RB51 and the mutant. Significant difference ( $\mathrm{P}$-value of $<0.05$ ) was calculated by $\mathrm{t}-$ test analysis. Results are expressed as the mean $+2 \mathrm{SD}$ of $\mathrm{X}$ individual mice per group $(0,7.14,21$ days after inoculation).

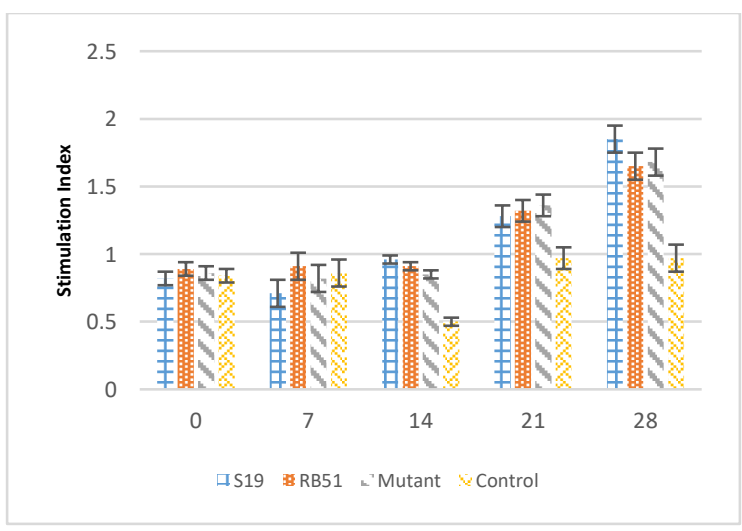

Figure 2. Average optical density obtained in the cell proliferation assay performed to evaluate immune responses in the mice during four weeks after immunization with Brucella abortus S19, RB51 and the mutant. Significant difference ( $P$-value of $<0.05$ ) was calculated by ttest analysis. Results are expressed as the mean $+2 \mathrm{SD}$ of $\mathrm{X}$ individual mice per group $(0,7.14,21,28$ days after inoculation).

Although the rate of proliferation in the mutantvaccinated group was not as high as that in the S19vaccinated group, it was higher than that in the RB51vaccinated group. These results indicated that vaccination with $\Delta w b k A$ is able to induce a cellular proliferative response. There was no significant difference between S19 cell stimulation and mutant strain, which demonstrated immunity suppression was not happened by deletion the mentioned gene.

\subsection{Statistical Analysis}

These results indicated the significant deference between S19-vaccinated and S19 $\Delta w b k A$-vaccinated groups in the levels of serum IgM, which represented reduction of the immunoglobulin stimulation by $\mathrm{S} 19$ $\triangle w b k A$ in comparison with S19. Also significant deference in S19-vaccinated, RB51-vaccinated and S19 $\triangle w b k A$ - vaccinated groups in cellular proliferative response was unremarkable. The confidence interval considered as $\mathrm{P}<0.05$.

\section{Discussion}

Brucellosis is a zoonotic transmissible disease that shows a high degree of morbidity, both in animals and humans. Consequently, it causes significant financial losses and represents a serious public health problem in many countries. Due to the serious economic losses and public health risk, extensive efforts have been made to prevent the disease in animals through vaccination programs (14). Live attenuated vaccines have been developed and successfully used against bovine brucellosis worldwide (15). B. abortus strain 19 is a smooth but attenuated strain. Strain 19 was quite effective in protecting cattle against subsequent infection with virulent strains of B. abortus (10). However, S19 has several problems that limit its use in cattle. In protective studies, it was discovered that the presence of smooth LPS in S19 interferes with the discrimination between infected and vaccinated animals during immune screening procedures (16). In order to avoid these problems, several strategies have been described for the development of alternative vaccines. One of them is the isolation of avirulent or attenuated strains lacking the $\mathrm{O}$-antigen (rough strains). These strains are unable to induce antibodies that interfere with the diagnosis (6).

The goal of this study was to eliminate defects of B.abortus S19 for the first time and obtain attenuated strains capable of inducing cellular immunity and protection but no antibody response against LPS in order to avoid interference with serological diagnosis was observed, the strains would ideally be genetically defined, preferably a deletion mutant with no antibiotic resistance and stable under field conditions.

The WbkA protein can interact with WboA to elongate the Brucella LPS O-side chain by $\alpha-1,2$ and $\alpha-1,3$ links. An early study has suggested that in $B$. abortus a $w b k A$ homologous gene is absolutely required for the $\mathrm{O}$-side chain production (11). In this work, we described the construction of a B. abortus glycosyltransferase ( $W b k A)$ insertion mutant in vaccine strain and its protection 
properties in mice. Althogh Zhi-qiang Li, et al suggested that native $2308 \Delta \mathrm{wbkA}$ is a potential attenuated vaccine against $16 \mathrm{M}$, this vaccine will be further evaluated in sheep (17).

Another study reported that rough wbkA B. abortus mutant is attenuated and a better vaccine than RB51 in $\mathrm{BALB} / \mathrm{c}$ mice. The preceding results suggest that the simultaneous vaccination with live $S$ avirulent $b v r S$ or $b v r R$ and the $\mathrm{R}$ attenuated $w b k A$ mutants is an interesting approach to improve the vaccine efficacy in the natural hosts, provided the serological interference was not considerable. As expected, the vaccination with the $\mathrm{R} w b k A$ mutant did not induce antibodies against the B. abortus LPS (18).

Lacerda et al. have demonstrated that B. abortus $\Delta w b k C$ mutants were attenuated in bone marrow derived macrophages could not reach a replicative niche and induced higher levels of interleukin-12 and tumor necrosis factor alpha compared to parental smooth strains. Additionally, the mutants exhibited attenuation in vivo in $\mathrm{C} 57 \mathrm{BL} / 6$ mice and interferon regulatory factor-1 knockout mice. $\Delta w b k C$ mutant strains induced lower protective immunity in C57BL/6 mice than that induced by smooth vaccine $S 19$ but similar to that induced by rough vaccine RB51 (19).

Overlap extension PCR is relatively straightforward, efficient and reliable method for correction and deletion of nucleotides and single point mutations, which can be accomplished by two rounds of PCR. Compared to alternative immunoassays, ELISA is the most versatile method, and the results are available within a short period of time. It also has the advantage of being readily automated, thus enabling its use as a screening test with the results numerically quantifiable. The presence of IgM antibodies specific for $B$. abortus in the serum samples of mice was determined by ELISA. An IgM response was elicited after infection with $B$. abortus S19 but not after infection with B. abortus S19 $\Delta w b k A$ or RB51. Therefore, these data indicate that after the use of B. abortus S19 $\triangle w b k A$ mutant for vaccination, interference with the diagnosis would be minimized.

The MTT assay is now widely accepted as a reliable test to examine cell proliferation. Although this work reported the inability of $B$. abortus S19 $\Delta w b k A$ attenuated mutant to induce IgM antibodies response, the results of the cell proliferation, evaluated by the MTT test, indicated that the stimulation with the mutant strain was sufficient to promote an adequate cell-mediated immune response, which is essential for controlling intracellular pathogens, as S19_per mutant mediates stronger cell mediated immuneresponse and more susceptible to polymyxin B andcomplement mediated killing (20).

\section{Conclusions}

The results of this study demonstrated that B. abortus S19 $\Delta w b k A$ does not induce IgM detectable antibody responses in ELISA. No significant difference between cell stimulation by available vaccine strains and mutant strain, not being rifampicin-resistance and reducing the existence mistakes to discriminate between vaccinated and infected animal, were the main advantages of the mutant generated in this study. Furthermore, the identity of the attenuation is known for the mutant, while still not completely known for RB51. However, due to this moderate protection efficacy, S19 $\Delta w b k A$ has to be further evaluated.

\section{Acknowledgements}

The authors wish to acknowledge the cooperation of the assistance of Veterinary Faculty of Tehran University and National Institute of Genetic Engineering and Biotechnology. Moreover, some parts of this research were financially supported by Iran National Science Foundation (Project No. 91004296).

\section{References}

1. Godfroid J, Cloeckaert A, Liautard JP, Kohler S, Fretin $\mathrm{D}$, Walravens $\mathrm{K}$, et al. From the discovery of the Malta fever's agent to the discovery of a marine mammal reservoir, brucellosis has continuously been a reemerging zoonosis. Vet Res. 2005;36(3):313-326. doi: 10.1051/vetres:2005003 pmid: 15845228

2. Adams LG. The pathology of brucellosis reflects the outcome of the battle between the host genome and the Brucella genome. Vet Microbiol.2002;90(1-4):553-561. doi: $\quad 10.1016 / s 0378-1135(02) 00235-3$ pmid: 12414171

3. Cardoso PG, Macedo GC, Azevedo V, Oliveira SC. Brucella spp noncanonical LPS: structure, biosynthesis, and interaction with host immune system. Microb Cell Fact. 2006;5:13. doi: 10.1186/1475-2859-5-13 pmid: 16556309

4. Conde-Alvarez R, Arce-Gorvel V, Iriarte M, MancekKeber M, Barquero-Calvo E, Palacios-Chaves L, et al. The lipopolysaccharide core of Brucella abortus acts as a shield against innate immunity recognition. PLoS Pathog. 2012;8(5):e1002675. doi: 10.1371/journal.ppat.1002675 pmid: 22589715

5. Zygmunt MS, Blasco JM, Letesson JJ, Cloeckaert A, Moriyon I. DNA polymorphism analysis of Brucella lipopolysaccharide genes reveals marked differences in O-polysaccharide biosynthetic genes between smooth and rough Brucella species and novel species-specific markers. BMC Microbiol. 2009;9:92. doi: 10.1186/1471-2180-9-92 pmid: 19439075

6. Olsen SC. Recent developments in livestock and wildlife brucellosis vaccination. Rev Sci Tech. 2013;32(1):207-217. doi: 10.20506/rst.32.1.2201 pmid: 23837378

7. Crasta OR, Folkerts O, Fei Z, Mane SP, Evans C, Martino-Catt $S$, et al. Genome sequence of Brucella abortus vaccine strain $S 19$ compared to virulent strains yields candidate virulence genes. PLoS One. 2008;3(5):e2193. doi: 10.1371/journal.pone.0002193 pmid: 18478107

8. Zhu J, Larson CB, Ramaker MA, Quandt K, Wendte JM, $\mathrm{Ku} \mathrm{KP}$, et al. Characterization of recombinant $\mathrm{B}$. abortus strain RB51SOD toward understanding the uncorrelated innate and adaptive immune responses 
induced by RB51SOD compared to its parent vaccine strain RB51. Front Cell Infect Microbiol. 2011;1:10. doi: 10.3389/fcimb.2011.00010 pmid: 22919576

9. Vemulapalli R, He Y, Buccolo LS, Boyle SM, Sriranganathan N, Schurig GG. Complementation of Brucella abortus RB51 with a functional wboA gene results in $\mathrm{O}$-antigen synthesis and enhanced vaccine efficacy but no change in rough phenotype and attenuation. Infect Immun. 2000;68(7):3927-3932. doi: 10.1128/iai.68.7.3927-3932.2000 pmid: 10858205

10. Caporale V, Bonfini B, Di Giannatale E, Di Provvido A, Forcella S, Giovannini A, et al. Efficacy of Brucella abortus vaccine strain RB51 compared to the reference vaccine Brucella abortus strain 19 in water buffalo. Vet Ital. 2010;46(1):13-19, 15-11. pmid: 20391363

11. Monreal D, Grillo MJ, Gonzalez D, Marin CM, De Miguel MJ, Lopez-Goni I, et al. Characterization of Brucella abortus O-polysaccharide and core lipopolysaccharide mutants and demonstration that a complete core is required for rough vaccines to be efficient against Brucella abortus and Brucella ovis in the mouse model. Infect Immun. 2003;71(6):3261-3271. doi: 10.1128/iai.71.6.3261-3271.2003 pmid: 12761107

12. Nayeri Fasaei B, Zahraei Salehi T, Naserli S, Saeedinia AR, Behroozikhah AM. Site-directed mutagenesis in Brucella abortus S19 by overlap extension PCR-based procedure. Journal of the Hellenic Veterinary Medical Society. 2018;68(3):273. doi: 10.12681/jhvms.15468

13. Arenas-Gamboa AM, Ficht TA, Kahl-McDonagh MM, Rice-Ficht AC. Immunization with a single dose of a microencapsulated Brucella melitensis mutant enhances protection against wild-type challenge. Infect Immun. 2008;76(6):2448-2455. doi: 10.1128/IAI.00767-07 pmid: 18362129
14. Barbier T, Nicolas C, Letesson JJ. Brucella adaptation and survival at the crossroad of metabolism and virulence. FEBS Lett. 2011;585(19):2929-2934. doi: 10.1016/j.febslet.2011.08.011 pmid: 21864534

15. Avila-Calderon ED, Lopez-Merino A, Sriranganathan $\mathrm{N}$, Boyle SM, Contreras-Rodriguez A. A history of the development of Brucella vaccines. Biomed Res Int. 2013;2013:743509. doi: 10.1155/2013/743509 pmid: 23862154

16. Corbel MJ. Brucellosis in humans and animals. Geneva: World Health Organization; 2006.

17. Li ZQ, Gui D, Sun ZH, Zhang JB, Zhang WZ, Zhang H, et al. Immunization of $\mathrm{BALB} / \mathrm{c}$ mice with Brucella abortus 2308DeltawbkA confers protection against wild-type infection. J Vet Sci. 2015;16(4):467-473. doi: 10.4142/jvs.2015.16.4.467 pmid: 26040616

18. Grillo MJ, Manterola L, de Miguel MJ, Munoz PM, Blasco JM, Moriyon I, et al. Increases of efficacy as vaccine against Brucella abortus infection in mice by simultaneous inoculation with avirulent smooth bvrS/bvrR and rough wbkA mutants. Vaccine. 2006;24(15):2910-2916.

10.1016/j.vaccine.2005.12.038 pmid: 16439039

19. Lacerda TL, Cardoso PG, Augusto de Almeida L, Camargo IL, Afonso DA, Trant CC, et al. Inactivation of formyltransferase (wbkC) gene generates a Brucella abortus rough strain that is attenuated in macrophages and in mice. Vaccine. 2010;28(34):5627-5634. doi: 10.1016/j.vaccine.2010.06.023 pmid: 20580469

20. Lalsiamthara J, Gogia N, Goswami TK, Singh RK, Chaudhuri P. Intermediate rough Brucella abortus S19Deltaper mutant is DIVA enable, safe to pregnant guinea pigs and confers protection to mice. Vaccine. 2015;33(22):2577-2583. doi: 10.1016/j.vaccine.20 15.04.004 pmid: 25869887 